\title{
Associação entre cognição e força de preensão manual em idosos: revisão integrativa
}

\author{
The association between cognition and handgrip strength \\ among the elderly: an integrative review
}

Nathalie Silva ${ }^{1}$

Tarciana Nobre de Menezes ${ }^{1}$

${ }^{1}$ Conselho de Ensino, Pesquisa e Extensão, Universidade Estadual da Paraíba. Av. das Baraúnas 351, Campus Universitário. 58109-753 Campina Grande PB Brasil. nathaliegmr@yahoo.com.br
Abstract The objective of this study is to identify researches that associated cognition and handgrip strength among the elderly. This is a bibliographic review, based on an integrative approach of articles published in the last five years, indexed in the PubMed, Lilacs and Scopus databases. Inclusion criteria were: observational or experimental studies with a sample of elderly people (aged 60 years or more); assessment of muscular strength using a manual dynamometer; seniors who had at least one cognitive assessment instrument. At the end of the search 10 articles were selected to examine cognitive function and statistical results in the sample. It was observed that most of the research was conducted among the elderly aged over 75 and the Mini Mental State Examination (MMSE) was identified as the scale most commonly used for global cognitive assessment. A significant association was identified between alterations in cognition and reduction of handgrip strength (HGS) in 90\% of the articles included in this study. Results of this review suggest the influence of cognitive impairment on the muscular strength of the elderly, which can affect aspects of their functional capacity and consequent dependence.

Key words Cognition, Muscular strength, Handgrip strength, Elderly
Resumo O objetivo deste estudo é identificar pesquisas que associaram a cognição com a força de preensão manual em idosos. Trata-se de uma revisão bibliográfica, tendo como base a abordagem integrativa, de artigos publicados nos últimos cinco anos, indexados nas bases de dados PubMed, Lilacs e Scopus. Os critérios de inclusão foram: estudos observacionais ou experimentais, com amostra composta por idosos (idade igual ou superior a 60 anos); avaliação da força muscular por meio de dinamometria manual; que apresentassem pelo menos um instrumento de avaliação cognitiva. Ao final da busca foram selecionados 10 artigos, caracterizados quanto à amostra, função cognitiva e resultados estatísticos. Observouse que a maior parte das pesquisas foi realizada em idosos com idade igual ou superior a 75 anos e identificou-se que o Mini Exame do Estado Mental (MEEM) foi a escala mais utilizada para avaliação cognitiva global. Verificou-se associação significativa entre alterações cognitivas e redução da força de preensão manual (FPM) em 90\% dos artigos desta revisão. Os resultados desta revisão sugerem a influência de alterações cognitivas na força muscular de idosos, podendo, assim, afetar aspectos de sua capacidade funcional e, consequentemente, dependência.

Palavras-chave Cognição, Força muscular, Força da mão, Idoso 


\section{Introdução}

No Brasil, nos últimos anos, houve aumento crescente no número de idosos, o que, aliado a maior expectativa de vida, representa avanço para a sociedade $^{1}$. No entanto, o envelhecimento só pode ser considerado como real conquista na medida em que se agregue qualidade aos anos adicionais de vida ${ }^{2}$, uma vez que o desenvolvimento econômico e a necessidade de políticas públicas são fortemente afetados pelas condições de saúde e autonomia do idoso ${ }^{3}$.

O envelhecimento favorece a redução da reserva fisiológica em diferentes sistemas, acarretando diminuição da capacidade de gerar respostas adaptativas frente a estímulos externos ${ }^{4}$. Nesse sentido, contribui para perda progressiva do desempenho físico e alterações do estado cognitivo do idoso, expondo-o, assim, a maior vulnerabilidade ${ }^{5,6}$.

Alterações na coordenação nervosa e no sistema musculoesquelético influenciam na função neuromuscular, contribuindo para a diminuição da condução nervosa e da força muscular ${ }^{7,8}$. A função muscular vem sendo utilizada como um dos indicadores de funcionalidade em idosos, tendo em vista que pode diminuir até atingir um nível cuja fraqueza passa a restringir a capacidade de realizar atividades cotidianas ${ }^{9}$.

Embora haja diferentes técnicas, a força de preensão manual (FPM) é recomendada como medida para mensuração de força muscular em idosos $^{10}$, por apresentar correlação com a força muscular total, não exigir grande esforço físico e estar relacionada com o desempenho físico ${ }^{11-13}$.

O envelhecimento está relacionado, ainda, com alterações no desempenho cognitivo do ido$\mathrm{so}^{5}$. Estudo populacional, na China ( $\geq 55$ anos), observou prevalência de $12,2 \%$ de comprometimento cognitivo entre os indivíduos avaliados ${ }^{14}$, enquanto no Reino Unido, em estudo populacional com idosos mais velhos ( $\geq 75$ anos), este foi de cerca de $50 \%$, sendo as maiores taxas observadas em idades mais avançadas ${ }^{15}$.

O comprometimento do desempenho cognitivo acarreta perda de autonomia e de independência do idoso, aumentando a carga de trabalho do cuidador e da família, e exigindo maior assistência dos serviços de saúde ${ }^{16}$. Portanto, avaliar a função cognitiva é fator importante no que se refere à saúde do idoso, possibilitando o diagnóstico precoce e o acompanhamento de demências ${ }^{17}$.

Alterações na função cognitiva parecem ter relação com o desempenho físico do idoso ${ }^{5,6}$. Um estudo nos Estados Unidos observou que idosos com declínio em funções como atenção e velocidade de processamento também apresentaram prejuízos no desempenho de habilidades como força de preensão e marcha ${ }^{18}$. Outro estudo com idosos, na China, observou que déficits de cognição estavam relacionados com FPM ruim, independentemente do ajuste por outras variáveis (idade, massa muscular, presença de morbidades e nível de atividade física $)^{19}$.

Diante do exposto, ressalta-se a necessidade de maior conhecimento acerca das alterações cognitivas do idoso, bem como a influência destas na função física, em especial na força muscular, contribuindo para a formulação de ações em saúde pública voltadas à prevenção destas condições. Além de nortear intervenções específicas e individuais por parte de profissionais de saúde e procedimentos clínicos envolvidos na reabilitação e manutenção da saúde física e mental dessa população. Desse modo, este estudo buscou identificar pesquisas que associaram a cognição com a força de preensão manual em idosos.

\section{Metodologia}

Este estudo caracteriza-se como revisão bibliográfica, com abordagem integrativa. Optou-se pela realização de uma revisão integrativa por ser um método de natureza ampla para obtenção, identificação, análise e síntese da literatura, por meio de achados provenientes de estudos primários, desenvolvidos mediante diferentes desenhos de pesquisa (permitindo a inclusão simultânea de estudos experimentais e observacionais). Desse modo, auxiliando a compreensão dos fenômenos e a ampliação dos conhecimentos sobre determinado tema. Portanto, esse método vem sendo muito utilizado em pesquisa na área de saúde 20,21 $^{\text {. }}$.

A revisão integrativa compreende de cinco etapas: 1) estabelecimento do problema, ou seja, definição do tema da revisão em forma de questão ou hipótese primária; 2) seleção da amostra (após definição dos critérios de inclusão); 3) caracterização dos artigos (definição das características ou das informações a serem coletadas dos artigos, por meio de critérios claros, norteados por instrumentos); 4) análise dos resultados (identificando similaridades e conflitos); e 5) apresentação e discussão dos achados ${ }^{20}$.

A busca eletrônica foi realizada no período de janeiro de 2015, nas bases de dados: PubMed (National Library of Medicine), Scopus (Elsevier) e Lilacs (Literatura Latino-americana e do Caribe 
em Ciências da Saúde). Foram selecionados artigos, publicados nos últimos cinco anos (2010 a 2014), nos idiomas inglês e português. Para a prospecção dos estudos, foram utilizados os descritores de forma combinada por meio dos operadores booleanos (AND e OR): cognition AND hand strength AND elderly OR aging OR older adults, e seus correspondentes em português: cognição AND força da mão AND idoso.

Para o cômputo do total de estudos foi verificada a duplicação ou triplicação dos mesmos entre as bases de dados, sendo cada artigo contabilizado somente uma vez. A partir dos estudos identificados, foram selecionados aqueles que preenchiam os critérios para sua inclusão considerando a leitura dos títulos e resumos. Foram selecionados somente os artigos que atenderam aos seguintes critérios de inclusão: estudos observacionais ou de intervenção, com amostra composta por idosos (idade igual ou superior a 60 anos); avaliação da força muscular por meio de dinamometria manual; que apresentassem pelo menos um tipo de avaliação cognitiva. Foram excluídos: artigos de revisão; estudos com: animais; idosos em reabilitação ou hospitalizados, com limitações articulares ou musculares, com doença neurológica e aqueles institucionalizados.

A coleta de dados foi feita sobre os estudos incluídos. As informações selecionadas para a ca- racterização dos estudos foram: autor, ano de publicação, local do estudo, tipo de estudo, amostra (idade, sexo), instrumento para avaliação da função cognitiva e resultados estatísticos.

\section{Resultados}

Um resumo da busca eletrônica realizada no mês de janeiro de 2015, nas bases de dados selecionadas, é apresentado na Figura 1. Inicialmente foram identificados 153 artigos, dos quais 50 foram excluídos por estarem em duplicatas, permanecendo 103, os quais foram submetidos à análise dos títulos e dos resumos e verificação dos critérios de inclusão e exclusão. Destes, 15 foram lidos na íntegra, dos quais somente $10^{18,22-}$ ${ }^{30}$ preenchiam adequadamente todos os critérios de inclusão, sendo, assim, selecionados para esta revisão integrativa.

A descrição dos artigos que verificaram a associação entre cognição e FPM em idosos é apresentada no Quadro 1. Dos 10 artigos selecionados, todos são observacionais, sendo: seis transversais $^{18,22,23,25,26,30}$ e quatro de coorte en, $^{24-29}$. Foram identificados artigos dos Estados Unidos $^{18,22}$, Sri Lanka ${ }^{23}$, Itália ${ }^{24}$, França ${ }^{25}$, Brasil $^{26}$, China $^{28}$, Canadá ${ }^{29}$ e Holanda ${ }^{27,30}$. Seis estudos apresentaram amostras com média etária superior a

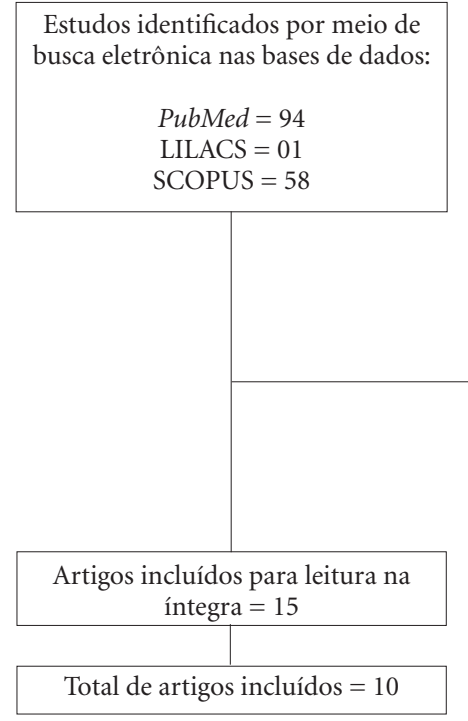

Estudos excluídos $=138$

Estudos em duplicata $=50$

Estudos não relacionados ao tema $=53$

Critérios de exclusão $=35$

- Idioma diferente do inglês ou português $=06$

- Amostra com indivíduos não idosos $=06$

- Idosos institucionalizados $=04$

- Idosos em reabilitação ou hospitalizados $=02$

- Idosos com limitações articulares/ musculares ou neurológicas $=13$

- Artigos de revisão = 04

Figura 1. Estudos incluídos e excluídos na revisão sobre associação entre cognição e força de preensão manual em idosos, de 2010 a 2014. 
Quadro 1. Características metodológicas dos estudos que avaliaram a associação da cognição e força de preensão manual em idosos, de 2010 a 2014.

\begin{tabular}{|c|c|c|c|c|}
\hline $\begin{array}{l}\text { Autor (ano), } \\
\text { local do estudo }\end{array}$ & $\begin{array}{l}\text { Tipo de } \\
\text { estudo }\end{array}$ & $\begin{array}{l}\text { Tamanho da } \\
\text { amostra (média } \\
\text { etária ou idade; } \\
\text { sexo) }\end{array}$ & $\begin{array}{c}\text { Instrumento de } \\
\text { avaliação: função } \\
\text { cognitiva }\end{array}$ & $\begin{array}{l}\text { Resultados } \\
\text { Estatísticos }\end{array}$ \\
\hline $\begin{array}{l}\text { Padubidri et al. }(2014)^{22} \text {, } \\
\text { Estados Unidos }\end{array}$ & Transversal & $\begin{array}{l}1.119 \text { idosos } \\
(80,8 \text { anos; } \\
\text { ambos os sexos })\end{array}$ & $\begin{array}{l}\text { MEEM: função } \\
\text { cognitiva global }\end{array}$ & $\begin{array}{l}\text { Idosos com menor declínio } \\
\text { cognitivo apresentaram } \\
\text { melhores valores de FPM } \\
(\beta=0,05 ; p=0,01) \text {. }\end{array}$ \\
\hline $\begin{array}{l}\text { Ukegbu et al. }(2014)^{23} \text {, } \\
\text { Sri Lanka }\end{array}$ & Transversal & $\begin{array}{l}252 \text { idosos }(71,1 \\
\text { anos; ambos os } \\
\text { sexos) }\end{array}$ & $\begin{array}{l}\text { MoCA: função } \\
\text { cognitiva global }\end{array}$ & $\begin{array}{l}\text { Idosos com melhor cognição } \\
\text { apresentaram melhores valores } \\
\text { de FPM }(\beta=0,21 ; p<0,001) \text {. }\end{array}$ \\
\hline $\begin{array}{l}\text { Gallucci et al. }(2013)^{24} \text {, } \\
\text { Itália }\end{array}$ & $\begin{array}{l}\text { Coorte } \\
\text { (7 anos) }\end{array}$ & $\begin{array}{l}309 \text { idosos }(80,2 \\
\text { anos; ambos os } \\
\text { sexos) }\end{array}$ & $\begin{array}{l}\text { MEEM: função } \\
\text { cognitiva global }\end{array}$ & $\begin{array}{l}\text { Associação significativa de maior } \\
\text { valor na escala de cognição e } \\
\text { melhor FPM }(p<0,0001) \text {. }\end{array}$ \\
\hline $\begin{array}{l}\text { McGough et al. }(2013)^{18} \text {, } \\
\text { Estados Unidos }\end{array}$ & Transversal & $\begin{array}{l}201 \text { idosos }(84,2 \\
\text { anos, ambos os } \\
\text { sexos) }\end{array}$ & $\begin{array}{l}\text { ADAS-Cog: } \\
\text { função cognitiva } \\
\text { global } \\
\text { TMT-A: atenção } \\
\text { visual } \\
\text { TMT-B: função } \\
\text { executiva } \\
\text { WMS-R LMI: } \\
\text { memória } \\
\text { ADAS-Cog Word } \\
\text { Recall: memória }\end{array}$ & $\begin{array}{l}\text { Não foi observada relação } \\
\text { significativa das variáveis de } \\
\text { função cognitiva com a FPM. }\end{array}$ \\
\hline $\begin{array}{l}\text { Kan et al. } \\
(2013)^{25} \\
\text { França }\end{array}$ & Transversal & $\begin{array}{l}3.025 \text { idosos } \\
\text { ( } 80,5 \text { anos; } \\
\text { mulheres) }\end{array}$ & $\begin{array}{l}\text { SPMSQ: função } \\
\text { cognitiva global }\end{array}$ & $\begin{array}{l}\text { Idosos com déficit cognitivo } \\
\text { apresentaram piores valores } \\
\text { médios de FPM em relação } \\
\text { aos idosos com boa cognição } \\
(\mathrm{p}<0,01) .\end{array}$ \\
\hline $\begin{array}{l}\text { Yassuda et al. }(2012)^{26} \text {, } \\
\text { Brasil }\end{array}$ & Transversal & $\begin{array}{l}384 \text { idosos }(72,3 \\
\text { anos; ambos os } \\
\text { sexos) }\end{array}$ & $\begin{array}{l}\text { MEEM: função } \\
\text { cognitiva global } \\
\text { BCSB (três } \\
\text { testes): } \\
\text { - Teste de } \\
\text { Memória } \\
\text { - Fluência verbal } \\
\text { - Teste do } \\
\text { desenho do } \\
\text { relógio }\end{array}$ & $\begin{array}{l}\text { Associação significativa do } \\
\text { valor médio do MEEM com o } \\
\text { desempenho da FPM (OR 3,03; } \\
\text { IC95\%: 1,75-5,26; p < 0,001). } \\
\text { Não houve relação significativa } \\
\text { entre os testes da BCSB com a } \\
\text { FPM. }\end{array}$ \\
\hline $\begin{array}{l}\text { Taekema et al. }(2012)^{27} \text {, } \\
\text { Holanda }\end{array}$ & $\begin{array}{l}\text { Coorte } \\
\text { (4 anos) }\end{array}$ & $\begin{array}{l}307 \text { idosos (entre } \\
85 \text { e } 89 \text { anos; } \\
\text { ambos os sexos) }\end{array}$ & $\begin{array}{l}\text { MEEM: função } \\
\text { cognitiva global } \\
\text { Abbreviated } \\
\text { Stroop test Trial } \\
\text { 3: atenção } \\
\text { LDST: velocidade } \\
\text { de processamento } \\
\text { 12-PLT: memória }\end{array}$ & $\begin{array}{l}\text { Idosos com melhor desempenho } \\
\text { no MEEM }(\beta=-0,25 ; p<0,001) \text {, } \\
\text { Stroop }(\beta=0,03 ; p=0,001), \\
\text { LDST }(\beta=-0,16 ; p<0,001) \text {, } \\
\text { 12-PLT (recordatório imediato) } \\
\text { ( } \beta=-0,18 ; p<0,001) ; 12-\text { PLT } \\
\text { (recordatório tardio) }(\beta=-0,38 ; \\
p<0,001) \text { apresentaram menor } \\
\text { declínio de FPM. }\end{array}$ \\
\hline
\end{tabular}




\begin{tabular}{|c|c|c|c|c|}
\hline \multicolumn{5}{|l|}{ Quadro 1. continuação } \\
\hline $\begin{array}{l}\text { Autor (ano), } \\
\text { local do estudo }\end{array}$ & $\begin{array}{l}\text { Tipo de } \\
\text { estudo }\end{array}$ & $\begin{array}{c}\text { Tamanho da } \\
\text { amostra (média } \\
\text { etária ou idade; } \\
\text { sexo) }\end{array}$ & $\begin{array}{l}\text { Instrumento de } \\
\text { avaliação: função } \\
\text { cognitiva }\end{array}$ & $\begin{array}{l}\text { Resultados } \\
\text { Estatísticos }\end{array}$ \\
\hline $\begin{array}{l}\text { Auyeung et al. }(2011)^{28} \text {, } \\
\text { China }\end{array}$ & $\begin{array}{l}\text { Coorte } \\
(4 \text { anos })\end{array}$ & $\begin{array}{l}2.737 \text { idosos } \\
\text { (homens: } 71,6 \\
\text { anos; mulheres: } \\
71,5 \text { anos) }\end{array}$ & $\begin{array}{l}\text { MEEM: função } \\
\text { cognitiva global }\end{array}$ & $\begin{array}{l}\text { O declínio cognitivo foi } \\
\text { associado com menor FPM, em } \\
\text { homens }(p<0,01) \text { e mulheres } \\
(p<0,05) .\end{array}$ \\
\hline $\begin{array}{l}\text { MacDonald et al. } \\
(2011)^{29} \\
\text { Canadá }\end{array}$ & $\begin{array}{l}\text { Coorte } \\
(6 \text { anos })\end{array}$ & $\begin{array}{l}1.043 \text { idosos } \\
(71,3 \text { anos; } \\
\text { ambos os sexos })\end{array}$ & $\begin{array}{l}5 \text { testes } \\
\text { cognitivos: } \\
\text { - Fluid reasoning } \\
\text { - Memória de } \\
\text { trabalho } \\
\text { - Memória } \\
\text { episódica } \\
\text { - Memória } \\
\text { semântica } \\
\text { - Crystallized } \\
\text { ability }\end{array}$ & $\begin{array}{l}\text { Idosos com pior desempenho } \\
\text { nos testes de fluid reasoning ( } \mathrm{p} \\
<0,001) \text {; memória de trabalho } \\
(\mathrm{p}<0,001) \text {; memória episódica } \\
(\mathrm{p}<0,001) \text {; memória semântica } \\
(\mathrm{p}<0,001) \text { e crystallized ability } \\
(\mathrm{p}<0,001) \text { apresentaram maior } \\
\text { declínio de FPM. }\end{array}$ \\
\hline $\begin{array}{l}\text { Taekema et al. }(2010)^{30} \text {, } \\
\text { Holanda }\end{array}$ & Transversal & $\begin{array}{l}555 \text { idosos (com } \\
85 \text { anos; ambos } \\
\text { os sexos) }\end{array}$ & $\begin{array}{l}\text { MEEM: função } \\
\text { cognitiva global }\end{array}$ & $\begin{array}{l}\text { Idosos com menor declínio } \\
\text { cognitivo apresentaram } \\
\text { melhores valores de FPM } \\
(\beta=0,25 ; p<0,001)\end{array}$ \\
\hline
\end{tabular}

MEEM: Mini Exame do Estado Mental; FPM: força de preensão manual; MoCA = Montreal Cognitive Assessment; ADAS-Cog: The Alzheimer's Disease Assessment Scale-Cognitive Subscale; TMT: Trail Making Tests; WMS-R LMI: Wechsler Memory Scale-Revised Logical Memory I; SPMSQ: Short Portable Mental Status Questionnaire; BCSB: Brief Cognitive Screening Battery; LDST: Letter Digit Substitution Task; 12-PLT: 12-Picture Learning Test.

$80 \operatorname{anos}^{18,22,24,25,27,30}$ e apenas um foi direcionado exclusivamente ao sexo feminino ${ }^{25}$.

Todos os artigos incluídos na revisão utilizaram escalas como instrumentos para avaliar a cognição, sendo o Mini Exame do Estado Mental (MEEM) a mais utilizada, tendo sido encontrada em seis estudos $22,24,26-28,30$. Outras escalas foram utilizadas para avaliar atenção ${ }^{18,27}$, função executiva $^{18}$, velocidade de processamento ${ }^{27}$, memória $^{18,26,27,29}$, vocabulário ${ }^{29}$ e fluência verbal ${ }^{26}$.

Nove artigos (90\%) identificaram que idosos com déficit cognitivo apresentaram menores valores médios para FPM, sendo essa associação significativa ${ }^{22-30}$. Apenas um estudo, realizado nos Estados Unidos com idosos com idade igual ou superior a 75 anos, não observou associação significativa entre função cognitiva global e a FPM ${ }^{18}$.

Os artigos apresentaram resultados variados no que concerne à associação entre memória e FPM. Dois artigos observaram associação significativa de problemas de memória de curto e longo prazo $^{27}$, de trabalho; episódica; e semântica ${ }^{29} \mathrm{com}$ pior desempenho na FPM. Dois outros artigos, contudo, não observaram associação significativa entre déficit de memória e piores valores de FPM em idosos ${ }^{18,26}$.

Pesquisa realizada na Holanda verificou associação estatisticamente significativa dos níveis de atenção e velocidade de processamento na $\mathrm{FPM}^{27}$, em outra realizada no Canadá observouse associação significativa entre vocabulário e $\mathrm{FPM}^{29}$. Dois artigos, os quais avaliaram outras variáveis cognitivas (função executiva, atenção visual e fluência verbal) não verificaram associação das mesmas com a FPM ${ }^{18,26}$.

\section{Discussão}

A velhice é uma fase da vida que pode ser considerada longa. De acordo com dados do Instituto Brasileiro de Geografia e Estatística (IBGE), de 2015, no Brasil, após os 60 anos a expectativa de vida era em média 15,1 anos $^{31}$. Em alguns casos, 
esta pode chegar a mais de 40 anos, ou seja, uma fase que pode tornar-se mais longa que a infância e a adolescência juntas. Esse fato, aliado ao declínio funcional ao qual o idoso está exposto, faz dessa população um grupo diferente dos mais jovens, apresentando necessidades específicas ${ }^{3}$.

O aumento na prevalência de doenças neurodegenerativas, no idoso, é um fenômeno global ${ }^{17}$. O estado cognitivo no idoso pode variar entre o funcionamento adequado até o comprometimento grave, levando à ocorrência de demên$\mathrm{cia}^{14}$. As síndromes demenciais caracterizam-se pelo déficit progressivo da função cognitiva, e constituem-se, atualmente, como problema de saúde pública e importante indicador de saúde entre idosos, devido à sua associação com a morbimortalidade nessa população ${ }^{16}$.

O desempenho funcional também vem sendo considerado na avaliação de saúde deste grupo, tendo em vista que o declínio da função física pode ser importante indicador de fragilidade e dependência ${ }^{32}$. A força muscular, por exemplo, é indicador sensível de perdas fisiológicas relacionadas com o desempenho em atividades diárias ${ }^{33}$. Portanto, sua avaliação tem papel fundamental para a saúde do idoso, atuando como potencial indicador de indivíduos em risco de deficiências funcionais ${ }^{9}$.

Publicações do European Working Group on Sarcopenia in Older People (EWGSOP) ${ }^{10}$, em 2010, e do Asian Working Group for Sarcopenia (AWGS) $)^{34}$, em 2014, recomendam o uso da FPM como medida para avaliação de força muscular no idoso. Isso se deve ao fato de a FPM ser uma medida de obtenção simples, ser marcador de baixa mobilidade e massa muscular, e por referir relação linear com o déficit em atividades de vida diária ${ }^{10}$. A FPM reflete, ainda, a força máxima derivada da contração dos músculos da mão e tem boa relação com outros grupos musculares ${ }^{13}$. Além disso, é um preditor independente para declínio na capacidade funcional do idoso ${ }^{12}$.

Observando os estudos incluídos nesta revisão é possível notar que a maioria foi conduzida com idosos pertencentes a grupos etários mais velhos, com idade igual ou superior a $75 \operatorname{anos}^{18,22,24,25,29,30}$. A escolha de idosos mais velhos pode ser devido à maior prevalência e/ ou risco de fragilidade neste grupo, cuja idade igual ou superior a 75 anos parece ser um fator de risco independente ${ }^{24}$. Independentemente da presença ou não de doença neurológica, o idoso poderá apresentar perda progressiva de alguma função cognitiva, a qual se torna mais evidente por volta dos $70 \operatorname{anos}^{35}$, devido a mudanças no sistema nervoso central, ca- racterizadas, entre outras, pela atrofia cortical ${ }^{36}$, a qual influencia, principalmente, o funcionamento de áreas vinculadas a funções cognitivas ${ }^{17}$. Entre as alterações relacionadas ao déficit cognitivo, pode-se citar a perda de memória e perturbações na função executiva, como inibição, planejamento e velocidade de processamento ${ }^{6,17}$.

As principais áreas responsáveis pela função cognitiva são o lobo pré-frontal e as temporais ${ }^{17}$. Estudo utilizando ressonância magnética funcional observou que idosos apresentaram menor atividade e conectividade de regiões cerebrais envolvidas em tarefas cognitivas, quando comparados aos indivíduos mais jovens, o que resulta na alocação de recursos insuficientes para a execução de tarefas, como a memória de trabalho ${ }^{37}$.

Outro estudo avaliando a atividade cerebral durante a execução de funções como memória de trabalho verbal e espacial observou que, quando submetidos aos testes de memória, os idosos apresentavam ativação bilateral da área pré-frontal, enquanto nos indivíduos jovens essa ativação ocorria apenas no lado dominante. Essa parece ser uma ação compensatória, a fim de manter a execução das funções requeridas, nesse caso a memória ${ }^{38}$. Essa "insuficiência” do córtex préfrontal parece estar associada à deficiência de substância branca nessa região $0^{39}$, o que poderia explicar a redução do número de conexões no lobo frontal dominante, sendo necessário recrutamento de outras áreas. Contudo, essa ação compensatória não parece estar relacionada apenas ao recrutamento adicional de áreas corticais. Com a redução do número de fibras aferentes (substância branca) no cérebro do idoso, as fibras remanescentes aumentam o potencial sináptico, de modo a compensar o processo de degeneração celular e manter a atividade cerebra ${ }^{40}$.

Diferentes métodos têm sido utilizados para avaliação cognitiva no idoso, como testes de memória, linguagem e avaliação da cognição global ${ }^{17}$. Entre estes, a neuroimagem parece ter maior precisão em identificar e quantificar as áreas cerebrais envolvidas na função cognitiva ${ }^{41}$, bem como avaliar os possíveis processos compensatórios associados à manutenção da função cognitiva com o aumento da idade ${ }^{42}$. Contudo, o alto custo de técnicas de neuroimagem funcional e estrutural, faz com que estas sejam requeridas em casos de déficit cognitivo evidente, como método confirmatório ${ }^{43}$, dificultando e, muitas vezes, inviabilizando seu uso em pesquisas com grandes amostras.

Com o objetivo de verificar a capacidade cognitiva dos idosos, todas as pesquisas selecionadas 
nesta revisão utilizaram apenas escalas como instrumento de rastreio, sendo o MEEM a mais utilizada para mensuração da função cognitiva global $^{22,24,26-28,30}$. Em uma revisão sistemática, utilizando estudos de coorte, observou-se que a maior parte dos artigos utilizava pelo menos uma medida de cognição global (46\%) e alguma outra avaliação específica (43\%), como memória e função executiva. Verificou-se, ainda, que o MEEM foi a escala mais frequente para avaliação cognitiva global $(88 \%)^{44}$. Essa escala é uma das mais utilizadas, por conter informações referentes à memória episódica, orientação temporoespacial, memória de trabalho, nomeação, linguagem e capacidade de copiar imagens, possibilitando obter um escore final do comprometimento cognitivo ${ }^{22}$.

Alguns dos estudos incluídos nesta revisão utilizaram, ainda, escalas que visavam mensurar funções específicas, tais como: atenção ${ }^{18,26}$, função executiva ${ }^{18}$, velocidade de processamento $^{26} \mathrm{e}$ memória ${ }^{18,26,27,29}$, de modo a observar as possíveis funções cognitivas que possam ter relação mais direta com o desempenho físico em idosos, nesse caso, a força muscular. De acordo com Schelini ${ }^{45}$, existe uma tendência de que sejam utilizados diferentes testes ou instrumentos de diversas baterias para avaliar a cognição, de modo que sejam elencados os instrumentos mais sensíveis e com melhor relação com a população que está sendo investigada.

Os artigos, em sua maioria, observaram associação significativa do déficit cognitivo com piores valores de FPM em idosos ${ }^{22-30}$. Isso pode ser devido à complexidade da função motora, tal como a exigida para realizar o movimento de FPM. O envelhecimento está associado com alterações qualitativas e quantitativas no córtex motor e na medula espinhal ${ }^{46}$. Do ponto de vista funcional, estas alterações corticais modificam conexões nervosas, levando ao comprometimento da força muscular ${ }^{36}$.

Contudo, a função motora parece estar relacionada não apenas ao córtex motor, mas às outras áreas corticais. Funções cognitivas superiores desempenhadas pelo córtex frontal, tais como atenção, velocidade com a qual a informação é processada e a memória, parecem também ter influência sobre o movimento ${ }^{47,48}$.

Uma coorte de quatro anos na Holanda observou associação significativa da redução de memória de curto e longo prazo com pior desempenho na $\mathrm{FPM}^{27}$. No Canadá, em uma coorte com seis anos de seguimento, observou-se associação significativa entre redução da memória de trabalho, episódica e semântica, com maior declínio da $\mathrm{FPM}^{29}$. Outras funções cognitivas como níveis de atenção ${ }^{27}$, vocabulário ${ }^{29}$ e velocidade de processamento $^{27}$ apresentaram associação significativa com o desempenho da FPM.

Estudos têm observado relação de áreas corticais relativas à função motora (córtex motor; córtex sensório-motor; e áreas motoras suplementares $)^{49}$, do cerebelo ${ }^{44,50}$ e gânglios da base (putâmen) ${ }^{49}$ com áreas cerebrais responsáveis pela função cognitiva (córtex pré-frontal) para execução de atividades motoras tais como a força de preensão manual.

Estudo avaliando a atividade cortical por meio de neuroimagem observou um aumento na atividade do lobo frontal de idosos durante atividades motoras de coordenação. Além disso, as áreas do córtex pré-frontal permaneciam ativadas por mais tempo nos idosos em relação aos indivíduos jovens ${ }^{51}$. Outro estudo constatou que idosos que mantinham maior conexão do córtex motor (lado dominante) e do cerebelo com o córtex pré-frontal apresentaram melhor desempenho no teste de FPM ${ }^{49}$. Além disso, outros estudos observaram que idosos apresentam ativação bilateral do córtex sensório-motor durante o teste de força de preensão, e não apenas do lado dominante, como nos indivíduos mais jovens ${ }^{49,52}$. Isso pode ser devido à degeneração celular e às mudanças bioquímicas no cérebro, ocasionadas pelo envelhecimento, no qual se exige um recrutamento adicional de áreas corticais para auxiliar na execução de outras funções ${ }^{51}$.

A função cognitiva está relacionada com a manutenção e a aprendizagem de habilidades motoras $^{53}$. Com o envelhecimento torna-se necessária maior atenção no que concerne à capacidade do idoso em adaptar-se a alterações ambientais $^{4}$. Essa adaptação está relacionada à cognição, responsável pela produção do comando motor e pela compensação de perturbações causadas por estímulos externos ${ }^{53}$. Dessa forma, pode-se inferir que alterações cognitivas possam influenciar na capacidade motora do idoso, o que justificaria o pior desempenho no teste de FPM em idosos com déficit cognitivo.

Em uma coorte de quatro anos realizada na Holanda, que objetivou analisar a relação temporal entre desempenho cognitivo e FPM, observou-se que o melhor desempenho cognitivo estava associado significativamente a um declínio mais lento na FPM. Os mesmos resultados foram observados quando consideradas as funções de memória (curto e longo prazo), velocidade de processamento e atenção ${ }^{27}$. Desse modo, os autores concluíram que o declínio cognitivo estava 
relacionado ao início da fraqueza muscular. Resultados semelhantes foram observados na Itália, após sete anos de seguimento, em que indivíduos com melhor desempenho cognitivo apresentaram melhores valores de FPM em relação aqueles com cognição ruim ${ }^{24}$.

Contudo, outros estudos têm questionado a temporalidade da associação entre cognição e FPM, supondo que déficits na força muscular levariam a um declínio na função cognitiva, e não o contrário ${ }^{18,30}$. No Canadá, após seis anos de seguimento, observou-se associação significativa entre pior rendimento de marcadores biológicos, incluindo a força muscular, no desempenho em testes de memória (de trabalho, episódica e semântica) e de vocabulário ${ }^{29}$. Além disso, idosos com maior força muscular parecem ter uma taxa mais lenta de declínio cognitivo, sugerindo que o declínio da força muscular precede o déficit cognitivo, e não o contrário ${ }^{54}$.

Tendo em vista essa divergência de resultados, ainda não foi devidamente esclarecido qual dos fatores influencia o declínio do outro. Boyle et al. ${ }^{54}$ sugerem que múltiplos fatores poderiam estar relacionados ao déficit de força e cognição em idosos, inclusive tendo alguns em comum, como alterações no sistema nervoso supraespinhal, que podem influenciar os movimentos, bem como afetar funções cognitivas.

Diante do exposto, observa-se que a temática deste estudo tem relevância para a área de saúde, tendo em vista o vínculo da perda cognitiva com a força muscular em idosos. Contudo, novas investigações devem ser realizadas a fim de preencher algumas lacunas. Entre estas, aprofundar a investigação sobre a influência e a contribuição de funções cognitivas específicas, como velocidade de processamento e aprendizagem, além da capacidade de plasticidade cerebral do idoso, na força muscular. Recomenda-se, ainda, investigar a relação da cognição com a FPM para idosos mais velhos (maiores de 75 anos) por meio de estudos de coorte, a fim de demarcar as princi- pais implicações ocasionadas por alterações cognitivas no desempenho físico, tal como na força muscular, com o avanço da idade.

\section{Conclusões}

A partir dos estudos identificados nesta revisão foi possível observar que pesquisas avaliando a cognição têm sido direcionadas para idosos de grupos etários mais avançados, tendo em vista estarem mais expostos à fragilidade. As escalas foram o principal instrumento utilizado pelos estudos para avaliar a função cognitiva, possivelmente pela facilidade e pelo baixo custo em relação a outros instrumentos. A maioria dos estudos mostrou, ainda, a existência de associação entre a cognição e a força de preensão manual em idosos, indicando uma possível influência do déficit de funções cognitivas no bom desempenho da força muscular.

A avaliação da função cognitiva e do desempenho físico no idoso, em especial a força muscular, bem como suas possíveis relações, é temática de relevância para as áreas de saúde pública, gerontologia e geriatria, tendo em vista a diminuição da funcionalidade e o aumento da dependência, observados na população idosa. Assim, é possível incentivar o investimento e a alocação de recursos em programas de saúde pública voltados para a integridade da função cognitiva e física no idoso, de modo a favorecer a manutenção ou a melhora de ambas as funções; prevenir ou mesmo retardar a ocorrência de demências e de dependência funcional. Além disso, busca-se incentivar a intersetorialidade na atenção à saúde em geriatria e gerontologia como um eixo estruturador na formulação de estratégias em saúde pública, com base em evidências, de modo a garantir assistência integral no cuidado ao idoso, nos três níveis de atenção à saúde, e favorecer o envelhecimento bem sucedido.

\section{Colaboradores}

N Silva e T Menezes participaram igualmente de todas as etapas de elaboração do artigo. 


\section{Referências}

1. Pereira JK, Firmo JOA, Giacomin KC. Maneiras de pensar e agir de idosos frente às questões relativas à funcionalidade/incapacidade. Cien Saude Colet 2014; 19(8):3375-3384.

2. Veras R. Envelhecimento populacional contemporâneo: demandas, desafios e inovações. Rev Saude Publica 2009; 43(3):548-554.

3. Camarano AA. TD 1840 - Estatuto do Idoso: Avanços com Contradições. Rio de Janeiro: IPEA; 2013.

4. Qingwei R, Zhuowei Y, Ma C, Zhijun B, Jin L, Wei H. Cognitive frailty, a novel target for the prevention of elderly dependency. Ageing Res Rev 2015; 20:1-10.

5. Won H, Singh DK, Din NC, Badrasawi M, Manaf ZA, Tan ST, Tai CC2, Shahar S. Relationship between physical performance and cognitive performance measures among community-dwelling older adults. Clin Epidemiol 2014; 6:343-350.

6. Blankevoort CG, Scherder EJ, Wieling MB, Hortobágyi T, Brouwer WH, Geuze RH, van Heuvelen MJ. Physical predictors of cognitive performance in healthy older adults: a cross-sectional analysis. PLoS One 2013; 8(7):e70799.

7. Reid KF, Pasha E, Doros G, Clark DJ, Patten C, Phillips EM, Frontera WR, Fielding RA. Longitudinal decline of lower extremity muscle power in healthy and mobility-limited older adults: influence of muscle mass, strength, composition, neuromuscular activation and single fiber contractile properties. Eur J Appl Physiol 2014; 114(1):29-39.

8. Narici MV, Maffulli N. Sarcopenia: characteristics, mechanisms and functional significance. $\mathrm{Br}$ Med Bull 2010; 95:139-159.

9. Barbat-Artigas S, Rolland Y, Cesari M, Kan GA, Vellas B, Aubertin-Leheudre M. Clinical Relevance of Different Muscle Strength Indexes Functional Impairment in Women Aged 75 Years and Older. J Gentorol A BiolSci Med Sci 2013; 68(7):811-819.

10. Cruz-Jentoft AJ, Baeyens JP, Bauer JM, Boirie Y, Cederholm T, Landi F, Martin FC, Michel JP, Rolland Y, Schneider SM, Topinková E, Vandewoude M, Zamboni M; European Working Group on Sarcopenia in Older People. Sarcopenia: European consensus on definition and diagnosis: Report of the European Working Group on Sarcopenia in Older People. Age Ageing 2010; 39(4):412-423.

11. Alley DE, Shardell MD, Peters KW, McLean RR, Dam TT, Kenny AM, Fragala MS, Harris TB, Kiel DP, Guralnik JM, Ferrucci L, Kritchevsky SB, Studenski SA, Vassileva MT, Cawthon PM. Grip strength cutpoints for the identification of clinically relevant weakness. J Gerontol A Biol Sci Med Sci 2014; 69(5):559-566.

12. Sugiura Y, Tanimoto Y, Watanabe M, Tsuda Y, Kimura $\mathrm{M}$, Kusabiraki T, Kono K. Handgrip strength as a predictor of higher-level competence decline among community-dwelling Japanese elderly in an urban area during a 4-year follow-up. Arch Gerontol Geriatr 2013 57(3):319-324.

13. Norman K, Stobäus N, Gonzalez MC, Schulzke JD, Pirlich M. Hand grip strength: Outcome predictor and marker of nutritional status. Clinical Nutrition 2011; $30(2): 135-142$.
14. Feng L, Ng XT, Yap P, Li J, Lee TS, Håkansson K, Kua $\mathrm{EH}, \mathrm{Ng}$ TP. Marital Status and Cognitive Impairment among Community-Dwelling Chinese Older Adults: The Role of Gender and Social Engagement. Dement Geriatr Cogn Dis Extra 2014; 4(3):375-384

15. Docking RE, Fleming J, Brayne C, Zhao J, Macfarlane GJ, Jones GT, Cambridge City over-75s Cohort (CC75C) study collaboration. Pain reporting in older adults: the influence of cognitive impairment - results from the Cambridge City $>75$ Cohort study. Br J Pain 2014; 8(3):119-124.

16. Holz AW, Nunes BP, Thumé E, Lange C, Facchini LA. Prevalence of cognitive impairment and associated factors among the elderly in Bagé, Rio Grande do Sul, Brazil. Rev Bras Epidemiol 2013; 16(4):880-888.

17. Soares FC, Oliveira TCG, Macedo LDD, Tomás AM, Picanço-Diniz DLW, Bento-Torres J, Bento-Torres NV3, Picanço-Diniz CW. CANTAB object recognition and language tests to detect aging cognitive decline: an exploratory comparative study. Clin Interv Aging 2015; 10:37-48.

18. McGough EL, Cochrane BB, Pike KC, Logsdon RG, McCurry SM, Teri L. Dimensions of physical frailty and cognitive function in older adults with amnestic mild cognitive impairment. Ann Phys Rehabil Med 2013; 56(5):329-341.

19. Auyeung TW, Kwok T, Lee J, Leung PC, Leung J, Woo J. Functional decline in cognitive impairment--the relationship between physical and cognitive function. Neuroepidemiology 2008; 31(3):167-173.

20. Whittemore R, Knafl K. The integrative review: updated methodology. J Adv Nurs 2005; 52(5):546-553.

21. Souza MT, Silva MD, Carvalho R. Integrative review: what is it? How to do it? Einstein 2010; 8(1):102-106.

22. Padubidri A, Snih SA, Samper-Ternent R, Markides KS, Ottenbacher KJ, Raji MA. Falls and cognitive decline in Mexican Americans 75 years and older. Clin Interv Aging 2014; 9:719-726.

23. Ukegbu U, Maselko J, Malhotra R, Perera B, Østbye T. Correlates of Handgrip Strength and activities of daily living in elderly Sri Lankans. J Am Geriatr Soc 2014; 62(9):1800-1801.

24. Gallucci M, Mazzuco S, Ongaro F, Di Giorgi E, Mecocci P, Cesari M, Albani D, Forloni GL, Durante E, Gajo GB, Zanardo A, Siculi M, Caberlotto L, Regini C. Body mass index, lifestyles, physical performance and cognitive decline: the "Treviso Longeva (TRELONG)" study. J Nutr Health Aging 2013; 17(4):378-384.

25. Kan GA, Cesari M, Gillette-Guyonnet S, Dupuy C, Nourhashémi F, Schott AM, Beauchet O, Annweiler C, Vellas B, Rolland Y. Sarcopenia and cognitive impairment in elderly women: results from the EPIDOS cohort. Age Ageing 2013; 42(2):196-202.

26. Yassuda MS, Lopes A, Cachioni M, Falcao DV, Batistoni SS, Guimaraes VV, Neri AL. Frailty criteria and cognitive performance are related: data from the FIBRA study in Ermelino Matarazzo, São Paulo, Brazil. J Nutr Health Aging 2012; 16(1):55-61. 
27. Taekema DG, Ling CH, Kurrle SE, Cameron ID, Meskers CG, Blauw GJ, Westendorp RG, de Craen AJ, Maier AB. Temporal relationship between handgrip strength and cognitive performance in oldest old people. Age Ageing 2012; 41(4):506-512.

28. Auyeung TW, Lee JS, Kwok T, Woo J. Physical frailty predicts future cognitive decline - a four-year prospective study in 2737 cognitively normal older adults. $J$ Nutr Health Aging 2011; 15(8):690-694.

29. MacDonald SW, DeCarlo CA, Dixon RA. Linking biological and cognitive aging: toward improving characterizations of developmental time. J Gerontol B Psychol Sci Soc Sci 2011; 66(Supl. 1):59-70.

30. Taekema DG, Gussekloo J, Maier AB, Westendorp RG, de Craen AJ. Handgrip strength as a predictor of functional, psychological and social health. A prospective population-based study among the oldest old. Age Ageing 2010; 39(3):331-337.

31. Instituto Brasileiro de Geografia e Estatística (IBGE). Sintese dos Indicadores Sociais: Uma Análise das Condições de Vida da População Brasileira 2015. Rio de Janeiro: IBGE; 2015.

32. Israel NEN, Andrade OG, Teixeira JJV. A percepção do cuidador familiar sobre a recuperação física do idoso em condição de incapacidade funcional. Cien Saude Colet 2011; 16(Supl. 1):1349-1356.

33. Ribeiro LHM, Neri AL. Exercícios físicos, força muscular e atividades de vida diária em mulheres idosas. Cien Saude Colet 2012; 17(8):2169-2180.

34. Chen LK, Liu LK, Woo J, Assantachai P, Auyeung TW, Bahyah KS, Chou MY, Chen LY, Hsu PS, Krairit O, Lee JS, Lee WJ, Lee Y, Liang CK, Limpawattana P, Lin CS, Peng LN, Satake S, Suzuki T, Won CW, Wu CH, Wu SN, Zhang T, Zeng P, Akishita M, Arai H. Sarcopenia in Asia: consensus report of the Asian Working Group for Sarcopenia. J Am Med Dir Assoc 2014; 15(2):95-101.

35. Rabbit P. Changes in problem solving ability in old age. In: Birren JE, Schaie KW, editors. Handbook of the Psychology of Aging. New York: Van Nostrand Reinhold; 1977. p. 606-625.

36. Manini TM, Hong SL, Clark BC. Aging and muscle: a neuron's perspective. Curr Opin Clin Nutr Metab Care 2013; 16(1):21-26.

37. Sambataro F, Murty VP, Callicott JH, Tan HY, Das S, Weinberger DR, Mattay VS. Age-related alterations in default mode network: impact on working memory performance. Neurobiol Aging 2010; 31(5):839-852.

38. DeCarli C, Kawas C, Morrison JH, Reuter-Lorenz PA, Sperling RA, Wright CB. Session II: Mechanisms of age-related cognitive change and targets for intervention: neural circuits, networks, and plasticity. J Gerontol A Biol Sci Med Sci 2012; 67(7):747-753.

39. Tullberg M, Fletcher E, DeCarli C, Mungas D, Reed BR, Harvey DJ, Weiner MW, Chui HC, Jagust WJ. White matter lesions impair frontal lobe function regardless of their location. Neurology 2004; 63(2):246-253.

40. Daselaar SM, Iyengar V, Davis SW, Eklund K, Hayes SM, Cabeza RE. Less wiring, more firing: low-performing older adults compensate for impaired white matter with greater neural activity. Cereb Cortex 2015; 25(4):983-990.
41. Rosano C1, Aizenstein HJ, Newman AB, Venkatraman V, Harris T, Ding J, Satterfield S, Yaffe K; Health ABC Study. Neuroimaging differences between older adults with maintained versus declining cognition over a 10year period. Neuroimage 2012; 62(1):307-313.

42. Goh JO, Beason-Held LL, An Y, Kraut MA, Resnick $\mathrm{SM}$. Frontal function and executive processing in older adults: process and region specific age-related longitudinal functional changes. Neuroimage 2013; 69:43-50.

43. Allard M, Husky M, Catheline G, Pelletier A, Dilharreguy B, Amieva H, Pérès K, Foubert-Samier A, Dartigues JF, Swendsen J. Mobile technologies in the early detection of cognitive decline. PLoS One 2014; 9(12):e112197.

44. Clouston SA, Brewster P, Kuh D, Richards M, Cooper R, Hardy R, Rubin MS, Hofer SM. The dynamic relationship between physical function and cognition in longitudinal aging cohorts. Epidemiol Rev 2013; 35:33-50.

45. Schelini PW. Teoria das inteligências fluida e cristalizada: Início e evolução. Estudos de Psicologia 2006; 11(3):323-332.

46. Manini TM, Clark BC. Dynapenia and Aging: Na Update. J Gerontol A Biol Sci Med Sci 2012; 67A(1):28-40.

47. Abe M, Hanakawa T. Functional coupling underlying motor and cognitive functions of the dorsal premotor cortex. Behav Brain Res 2009; 198(1):13-23.

48. Olivier E, Davare M, Andres M, Fadiga L. Precision grasping in humans: from motor control to cognition. Curr Opin Neurobiol 2007; 17(6):644-648.

49. Seidler R, Erdeniz B, Koppelmans V, Hirsiger S, Mérillat $S$, Jäncke L. Associations between age, motor function, and resting state sensorimotor network connectivity in healthy older adults. Neuroimage 2015; 108:47-59.

50. Stoodley CJ, Valera EM, Schmahmann JD. Functional topography of the cerebellum for motor and cognitive tasks: an fMRI study. Neuroimage 2012; 59(2):15601570.

51. Heuninckx S, Wenderoth N, Debaere F, Peeters R, Swinnen SP. Neural basis of aging: the penetration of cognition into action control. J Neurosci 2005; 25(29):6787-6796.

52. Ward NS, Swayne OB, Newton JM. Age-dependent changes in the neural correlates of force modulation: an fMRI study. Neurobiol Aging 2008; 29(9):1434-1446.

53. Trewartha KM, Garcia A, Wolpert DM, Flanagan JR. Fast but fleeting: adaptive motor learning processes associated with aging and cognitive decline. J Neurosci 2014; 34(40):13411-13421.

54. Boyle PA, Buchman AS, Wilson RS, Leurgans SE, Bennett DA. Association of muscle strength with the risk of Alzheimer disease and the rate of cognitive decline in community-dwelling older persons. Arch Neurol 2009; 66(11):1339-1344

Artigo apresentado em 11/03/2015

Aprovado em 14/01/2016

Versão final apresentada em 16/01/2016 